\title{
HACIA UNA PROSODIA BASADA EN EL USO: ACTOS DE HABLA EN EL ESPAÑOL MEXICANO
}

\author{
TOWARDS A USAGE-BASED PROSODY: SPEECH ACTS IN MEXICAN SPANISH
}

\section{RESUMEN:}

Pedro Martín ButragueÑo

El Colegio de México

El trabajo muestra algunas aportaciones del estudio de la prosodia basado en el uso lingüístico. Esta prosodia se basa en entrevistas cara a cara, muestras representativas en diferentes comunidades, habla despreocupada, una visión constructiva de los procesos lingüísticos, datos analizados en contexto, etc. Permite la generación de hipótesis sobre el desarrollo lingüístico, el cambio y la variación y en general un análisis de corte más realista. A este propósito, se comparan enunciados aseverativos y directivos desde un punto de vista regulativo, y también se estudian enunciados expresivos desde una perspectiva constitutiva. Los datos se representan en términos de la llamada teoría de optimidad estocástica.

PALABRAS CLAVE: prosodia, actos de habla, español de México.

\section{ABSTRACT:}

This work shows some advances in the study of the linguistic usage-based prosody. This kind of prosody rests on face-to-face interviews, a representative sampling of speech in different communities, a constructive view of linguistic processes, data analyzed in context, and so on. It allows proposing a number of hypotheses about linguistic development, change and variation, and an analysis generally more realistic. According to this proposal, declarative and directive speech acts are compared from a regulative point of view, and also expressive speech acts are studied, but from a constitutive point of view. Data are represented following the principles of stochastic optimality theory.

KEY WORDS: prosody, speech acts, Mexican Spanish. 


\section{INTRODUCCIÓN}

El propósito de este artículo es mostrar algunos avances en el estudio de la prosodia de los actos de habla (Yule, 2011; Orozco, 2012) en el español mexicano ${ }^{1}$. El planteamiento descansa en varios supuestos: a) el desarrollo de una prosodia basada en el uso (PBU) como hilo conductor entre los datos y un planteamiento lingüístico general (Bybee, 2001, 2008; Bybee y Hopper, 2001; Tomasello, 2003; Martín Butragueño y Velásquez, Upegui 2014); b) la necesidad de acercamientos multivariables, amparados en la existencia de tres tipos de fenómenos: regulaciones, constituciones e instrucciones (Dittmar, 1996; Martín Butragueño, 2014a); c) la formalización de los datos en términos métrico-autosegmentales y optimales (Gussenhoven, 2004), con basamento en los hechos fonéticos (Martínez Celdrán, 2011; Murrieta, en preparación), al tiempo que de la forma más convencional posible (Hualde y Prieto, en preparación); d) la concepción dinámica de la prosodia, en su instrumentación por los hablantes y en su análisis.

La PBU busca ofrecer una perspectiva realista sobre los procesos lingüísticos (Martín Butragueño, 2014a). El término realista debe entenderse aquí desde al menos tres principios metodológicos: la representatividad social y discursiva, la ponderación del error ${ }^{2}$ y la exhaustividad (Labov, 1994, 2001, 2010). La PBU es análoga a la llamada gramática basada en el uso (Bybee, 2001, 2008) en supuestos y propósitos. Una PBU permite generar ciertas hipótesis: a) el carácter fonético-fonológico de una estructura prosódica depende de la cantidad de contexto necesario para describirla ( $c f$. Dittmar, 1996); b) la frecuencia de una estructura permite el asentamiento social, la negociación de identidades, el aprovechamiento conversacional y el aprendizaje apropiado (cf. Bybee y Hopper, 2001; Aguilar Ruiz, 2012); c) el sentido pragmático de la aportación prosódica a los enunciados se construye a partir del uso en un contexto dado, etc. La PBU es cercana en sus planteamientos metodológicos a los empleados en el estudio de la entonación coloquial (Hidalgo, 1997) o de la cortesía prosódica (Hidalgo, 2009, 2011; Orozco, 2010; y referencias en <http://fonocortesia.es/>), aunque con cierto énfasis en que no solo el discurso, sino también el sistema prosódico puede estudiarse desde el uso. Asimismo, la PBU se ha concebido metodológica $y$ teóricamente en un marco cercano a los estudios de variación y cambio, tomando las comunidades de práctica como como marco de referencia para la indización de significados sociales (Eckert, 2000, 2008), considerando la estratificación, el contacto,

\footnotetext{
${ }^{1}$ Agradezco la gentileza del prof. Antonio Hidalgo, al invitarme a leer una versión de este texto como conferencia plenaria en el VI Congreso Internacional de Fonética Experimental (Universitat de València, 6-8 de noviembre de 2014). Es parte del proyecto «Diversidad y variación fónica en las lenguas de México: Hacia una nueva caracterización de la diversidad geolingüística» (CONACYT, 127876). ). Al tiempo, se trata de un trabajo preparatorio para la Fonología variable del español de México. Vol. II: Prosodia enunciativa.

${ }^{2}$ Esta idea es general en el trabajo científico y suele atribuirse a Newton.
} 
el avance y retracción de fenómenos específicos (Martín Butragueño, 2014c) y las diferencias entre estilos contextuales ${ }^{3}$.

Dependiendo del papel del contexto, se hablará de regulaciones, constituciones e instrucciones ${ }^{4}$. Las regulaciones describen actividades y comportamientos independientes de las reglas; las constituciones se refieren a acciones que dependen de las reglas mismas; las instrucciones son formulaciones muy sensibles al contexto. Las regulaciones tienen la forma $S i X$, entonces $Y$, e incorporan poca cantidad de contexto. En una primera sección se discuten las regulaciones de modo local y global con algunos datos de lectura en pares mínimos representativos vs. directivos de pregunta absoluta procedentes de AMPER-México (tipo El órgano se toca con paciencia vs. ¿El órgano se toca con paciencia?). Las regulaciones, insertas en la tradición que plasma los modos de las ciencias naturales en las expresiones sociológicas, describen la relación del proceso dinámico con las categorías vinculadas a él; regulan las conductas, al expresar que ciertos comportamientos deben o pueden realizarse.

Una constitución tiene la forma En el contexto $Z$, x cuenta como $Y$. Por su parte, una instrucción se expresa como Encuentra una $z$ minúscula, y ve que en el contexto $z, x$ cuente como $Y$. Los actos de habla expresivos (infra) se han tratado como constituciones, aunque sus características sugieren que el trabajo posterior debería abordarlos como instrucciones (es decir, máximamente vinculados al contexto). Los actos expresivos (;Puros nacos!, ;A veces lloro mis lágrimas!) se han extraído de entrevistas sociolingüísticas de 36 hablantes y los resultados se han ordenado oponiendo expresiones de ánimo a expresiones de desánimo en los extremos pragmáticos. Las constituciones sirven para definir prácticas o instituciones sociales, al expresar cómo se llevan a cabo ciertos actos; sus contextos relativamente generales suelen necesitar de indización, para saber con exactitud a quién o qué se aplican. Las instrucciones necesitan introducir gran cantidad de información contextual para captar la naturaleza muy específica de la situación, y pueden llegar a ser circunstanciales.

La taxonomía que separa regulaciones, constituciones e instrucciones, aplicada a la prosodia, distingue la relación que puede darse entre enunciados, sea sintácticosemántica, pragmática o discursiva, estableciendo vínculos fonético-fonológicos de

${ }^{3}$ Sin entrar en muchos detalles acerca de la formalización, ésta se enmarca en la teoría de la optimidad (TO) (Prince y Smolensky, 2004 [1993]), y en especial en las versiones estocásticas (TOE) de esta perspectiva (Díaz Campos y Colina, 2006; Pater, 2009; Coetzee, 2009; Kostakis, 2010; Coetzee y Kawahara, 2013; Martín Butragueño, 2014a: 89-109; Serrano, 2014), entendiéndolas solo como un recurso descriptivo. La TOE regula el peso de las restricciones a través de algoritmos. Uno de los más conocidos es el algoritmo de aprendizaje gradual, de Boersma y Hayes (2001), pero no es el único. En los trabajos variacionistas se ha empleado la probabilidad logística (Guy, 1994, 1997, 2007) y existen algunas investigaciones que simplemente emplean medidas de frecuencia (Feldhausen y Vanrell, 2014). Los candidatos considerados se presentan todos en la comunidad de habla; las marcas de infracción $\left(^{*}\right)$ y respeto $(\mathcal{V})$ a una restricción se entienden como tendencias. Por la generalización estocástica de Guy (2007:26), se exponen las observaciones aceptando que unos mismos instrumentos sirven para los hechos categóricos y los variables. La formulación de las restricciones sigue de cerca o adapta planteamientos de Gussenhoven (2004).

${ }^{4}$ La adaptación y ejemplificación de los planteamientos de Dittmar (1996) a la organización de una fonología variable, pueden verse en detalle en Martín Butragueño (2014a: 55-61). 
diferentes niveles entre las producciones lingüísticas, y está en la base de una PBU, en la medida en que se parte de la idea de que el comportamiento lingüístico y social están íntimamente vinculados y se describen por los mismos tipos de reglas.

2. ENUNCIADOS ASEVERATIVOS VS. DIRECTIVOS (INTERROGATIVOS ABSOLUTOS): UN ENFOQUE REGULATIVO

La diferencia entre enunciados aseverativos e interrogativos, especialmente los absolutos, es uno de los problemas más estudiados en entonación del español (cf. Martínez Celdrán, 2011). Los enunciados aseverativos son propios de las aserciones de creencia y pensamiento, pero también habituales en fragmentos discursivos narrativos y descriptivos (Yule, 2011). En cuando a las preguntas, aun las que buscan solo información pueden considerarse peticiones, de hecho peticiones de información (Orozco, 2012), y son una subclase específica de los enunciados directivos.

La discriminación entre unos y otros enunciados se presta bien al tratamiento por medio de reglas regulativas, que necesitan un contexto mínimo para explicitar la aparición de cada variante. En términos optimales, las reglas regulativas tendrían su equivalente en jerarquizaciones restrictivas relativamente simples. El número de diferencias, sin embargo, es relativamente amplio y, además, no se producen de una manera estable, ni en casos específicos ni en el seno de la comunidad de habla.

Quisiera referirme a la representación fonético-fonológica de una serie de hallazgos fonéticos obtenidos en un trabajo previo (Martín Butragueño, en prensa), a partir de la comparación de pares mínimos en estilo de lectura, siguiendo el llamado Corpus 1 de AMPER. Las observaciones fonéticas son tanto locales como globales y se leen ahora desde la teoría de la optimidad (TO). La TO postula restricciones pretendidamente universales, que representan las distintas fuerzas lingüísticas en competencia a la hora de producir una solución sistemática; no son categóricas, sino que están jerarquizadas, siendo que algunas tienen un peso mayor que otras en algunas lenguas o en algunas variedades. Un ordenamiento estructural diferente puede producir un sentido pragmático diferente. Así, la restricción NO RISE puede infringirse si en una sílaba nuclear se introduce una prominencia, como un foco contrastivo o un foco informativo de dominio local, o respetada, si se no se introduce ${ }^{5}$. En los procesos de variación y cambio suelen entrar en competencia las restricciones de FIDELIDAD (que producen soluciones más conservadoras) y las de BUENA FORMACIÓN, bien sea ésta contextual o intrínseca. Al hablar de prosodia, es necesario referirse al menos a dos o tres familias más de restricciones: las de ASOCIACIÓN, las de ALINEAMIENTO y las de FRASEO (Gussenhoven, 2004).

La tabla 1 muestras las diferencias locales y la tabla 2 las globales. En la columna de la izquierda se anota la pista fonética considerada, en la del centro la generalización encontrada en los datos (habitualmente acompañada de un dato cuantitativo), y en la de

\footnotetext{
${ }^{5}$ Hay variedades, como ocurre en el habla popular mexicana, en las que puede infringirse incluso aunque no esté presente un foco en tal posición.
} 
la derecha la posible restricción. A veces se anota entre paréntesis una referencia al código biológico general en que podría encuadrarse la restricción.

\begin{tabular}{|c|c|c|}
\hline Pista fonética & Generalización sobre los resultados & Posible restricción \\
\hline $\begin{array}{l}\text { Altura del pico } \\
\text { inicial }\end{array}$ & $\begin{array}{l}\text { Solo el } 27,7 \% \text { de las comparaciones } \\
\text { muestran Dir }>\text { Rep con } 1,5 \text { st o más de } \\
\text { diferencia }\end{array}$ & $\begin{array}{l}\text { PEAK SIZE } F / \sigma_{\mathrm{PN} 1}(\mathrm{Dir}) \\
\text { (Código de tamaño por frecuencia) }\end{array}$ \\
\hline Acento inicial & $\begin{array}{l}\text { En todos los casos de Rep y Dir aparecen } \\
\text { acentos bitonales ascendentes (LH). Solo en } \\
\text { el } 22,2 \% \text { se trata de un acento diferente (por } \\
\text { ejemplo L+>H* vs. } \mathrm{L}^{*}+\mathrm{H} \text { ) }\end{array}$ & NO RISE/ $\sigma_{\mathrm{PN} 1}($ Rep, Dir) \\
\hline Inicio absoluto & $\begin{array}{l}\text { Solo en el } 22,2 \% \text { de los pares Rep/Dir, Dir }> \\
\text { Rep con } 1,5 \text { st o más de diferencia }\end{array}$ & $\begin{array}{l}\text { HAVE BOUNDARY }(\mathrm{T})(\mathrm{Dir}) \\
\text { ALIGN }(\mathrm{L} / \mathrm{l}, \mathrm{izq})(\mathrm{Rep}) \\
\text { ALIGN }(\mathrm{H} / \mathrm{l}, \mathrm{izq})(\mathrm{Dir}) \\
\text { (Código de esfuerzo) }\end{array}$ \\
\hline Pico intermedio & $\begin{array}{l}\text { Solo en el } 16,6 \% \text { de las } 18 \text { ocasiones Dir }> \\
\text { Rep con una diferencia igual o mayor a } 1,5 \mathrm{st}\end{array}$ & $\begin{array}{l}\text { PEAK SIZE F/ } \sigma_{\mathrm{PN} 2}(\mathrm{Dir}) \\
\text { (Código de tamaño por frecuencia) }\end{array}$ \\
\hline $\begin{array}{l}\text { Altura del } \\
\text { acento nuclear }\end{array}$ & $\begin{array}{l}\text { En el } 83,3 \% \text { de los casos, Dir }>\text { Rep de } \\
\text { forma significativa }\end{array}$ & $\begin{array}{l}\text { PEAK SIZE F/ } \sigma_{\mathrm{N}} \text { (Dir) } \\
\text { (Código de tamaño por frecuencia) }\end{array}$ \\
\hline $\begin{array}{l}\text { Tendencia en el } \\
\text { movimiento del } \\
\text { acento nuclear }\end{array}$ & $\begin{array}{l}\text { Rep es descendente en el } 83,3 \% \text { de los } \\
\text { promedios, pero Dir es ascendente solo en el } \\
50 \% \text { de los casos. Solo en el } 33,3 \% \text { hay } \\
\text { diferencias claras entre ambos tipos de } \\
\text { enunciados }\end{array}$ & $\begin{array}{l}\text { NO RISE } / \sigma_{N}(\text { Rep }) \\
\text { NO FALL } / \sigma_{N}(\text { Dir })\end{array}$ \\
\hline $\begin{array}{l}\text { Tramo L en el } \\
\text { acento nuclear } \\
\text { como marca de } \\
\text { Rep }\end{array}$ & $\begin{array}{l}\text { El } 100 \% \text { de los casos de Rep muestran un L, } \\
\text { frente al } 50 \% \text { de los casos de Dir difieren, } \\
\text { pues, en el } 50 \% \text { de las ocasiones }\end{array}$ & $\begin{array}{l}\mathrm{L}^{*} \rightarrow \operatorname{TBU}\left(\sigma_{\mathrm{N}}^{*}\right)(\mathrm{Rep}) \\
\operatorname{ALIGN}\left(\mathrm{L} / \sigma_{\mathrm{N}}, \text { izq }\right)(\mathrm{Dir})\end{array}$ \\
\hline $\begin{array}{l}\text { Altura del tono } \\
\text { de juntura }\end{array}$ & $\begin{array}{l}\text { Dir es siempre significativamente superior a } \\
\text { Rep }\end{array}$ & $\begin{array}{l}\text { ALIGN (L/l, der) (Rep) } \\
\text { ALIGN (H/l, der) (Dir) } \\
\text { (Código de tamaño por frecuencia) }\end{array}$ \\
\hline $\begin{array}{l}\text { Tipo de tono de } \\
\text { juntura }\end{array}$ & $\begin{array}{l}\text { El tono de juntura es siempre diferente. Rep } \\
\text { es } \mathrm{L} \% \text { en el } 94,4 \% \text { de los casos; Dir es } \\
\text { siempre } \mathrm{H} \%\end{array}$ & $\begin{array}{l}\text { ALIGN (L/l, der) (Rep) } \\
\text { ALIGN (H/l, der) (Dir) } \\
\text { (Código de tamaño por frecuencia) }\end{array}$ \\
\hline $\begin{array}{l}\text { Duración de las } \\
\text { sílabas }\end{array}$ & $\begin{array}{l}\text { De las } 66 \text { medias de las sílabas prominentes, } \\
\text { Dir }>\text { Rep en el } 68,2 \% \text { de las ocasiones. En } \\
\text { general, la escala de duraciones o } \\
\text { prominencia temporal, es } \sigma_{\mathrm{N}}>\sigma_{\mathrm{PN} 1}>\sigma_{\mathrm{F}}> \\
\sigma_{\mathrm{PN} 2}\end{array}$ & $\begin{array}{l}\text { DURATION SIZE (Dir) } \\
\text { (Código de tamaño por duración) }\end{array}$ \\
\hline $\begin{array}{l}\text { Magnitud de la } \\
\text { intensidad }\end{array}$ & $\begin{array}{l}\text { Se postula }\left[[\operatorname{Rep} \geq \operatorname{Dir}]_{\mathrm{PN}}[\mathrm{Dir}>\operatorname{Rep}]_{\mathrm{N}}\right] \text {, pues } \\
\text { en el material prenuclear, la intensidad de R } \\
\text { es mayor en el } 61,1 \% \text { de las ocasiones; en la } \\
\text { sílaba nuclear y la juntura, Dir }>\text { Rep en el } \\
91,6 \% \text { de las veces }\end{array}$ & $\begin{array}{l}\text { INTENSITY } / \omega_{\mathrm{PNS}}(\text { Rep}) \\
\text { INTENSITY } / \omega_{\mathrm{N}}(\mathrm{Dir}) \\
\text { (Código de producción) }\end{array}$ \\
\hline $\begin{array}{l}\text { Ensordecimiento } \\
\text { vocálico }\end{array}$ & $\begin{array}{l}\text { De los } 81 \text { casos de ensordecimiento (en } 1188 \\
\text { vocales, el } 6,8 \% \text { ), el } 82,7 \% \text { apareció en } \\
\text { enunciados Rep, y solo el } 17,3 \% \text { en } \\
\text { enunciados Dir }\end{array}$ & HAVE NUC(son) (Dir) \\
\hline
\end{tabular}

Tabla 1. Diferencias fonéticas locales y posible restricción afectada 
Así, con respecto a la altura del pico inicial, la generalización es que ésta es mayor en los enunciados directivos; ahora bien, la diferencia entre ambos tipos de enunciados solo supera un umbral de 1,5 st el $27,7 \%$ de las veces ${ }^{6}$. En términos formales se propone una restricción de buena formación PEAK SIZE $\mathrm{F} / \sigma_{\mathrm{PN} 1}$, que pediría aumentar el pico en la primera sílaba prenuclear, con respecto a otros enunciados. Esta restricción sería respetada por los enunciados directivos e infringida por los representativos. Sería además una restricción de bajo nivel, pues solo estaría activa una cuarta parte de las veces; por eso en la tabla 3, que sintetiza el efecto de las restricciones, se sitúa en una columna del lado derecho. Para evitar el carácter ad hoc de la restricción, ésta se encuadra en la propuesta de códigos biológicos de Gussenhoven (2004, cap. 5) para el comportamiento prosódico, que será empleada para varios de los casos aquí expuestos, entendida como tendencias y no necesariamente con efectos en la gramaticalización; ésta última es factible sobre todo en el terreno informativo. Existirían tres códigos: a) el código de frecuencia (basado en Ohala, 1983, 1984, 1994), que con base en el tamaño fisiológico, opone lo alto y lo bajo y se interpreta lingüísticamente como la diferencia entre interrogaciones y aserciones, entre otros correlatos, como la incertidumbre y la certidumbre, al que aquí me referiré como código de tamaño, suponiendo plausible la generalización a todos los parámetros acústicos; b) el código de producción, que a partir de la idea de fases de energía atribuye el comienzo al grado alto y al final el grado bajo, de forma que por ejemplo inicios altos señalan tópicos nuevos, e inicios bajos continuación de tópicos (Lieberman, 1967; Gussenhoven, 2002, 2004), y c) el código de esfuerzo (de Jong, 1995; Gussenhoven, 2002, 2004), que basado en el nivel, correlaciona el mayor esfuerzo con el mayor movimiento y viceversa, de manera que una mayor excursión señala relevancia informativa, así como sorpresa y amabilidad en lo afectivo, que interviene por ejemplo en el tono de juntura inicial (como la 'servicialidad' en holandés, $c f$. Gussenhoven, 2004:94) y especialmente en la construcción del foco (véase Vanrell, Stella, Gili Fivela y Prieto 2013 a propósito del foco contrastivo en catalán, italiano y español $)^{7}$.

Además de a PEAK SIZE $F / \sigma_{\mathrm{PN} 1}$, el código de tamaño subyacería también a PEAK SIZE $\mathrm{F} / \sigma_{\mathrm{PN} 2}$, PEAK SIZE $F / \sigma_{\mathrm{N}}$ y DURATION SIZE. Las tres primeras se refieren a la altura tonal y la cuarta a la mayor duración; todas ellas son cumplidas por los enunciados directivos e infringidas por los representativos. Al igual que la restricción referida a la altura de la primera sílaba tónica, la de la segunda tónica se activa solo en un número limitado de ocasiones, como se indica en la tabla 1, por lo que ocupa un lugar bajo en la jerarquía de la tabla 3. La mayor duración temporal y, en especial, la altura del pico nuclear, en

\footnotetext{
${ }^{6}$ Se parte de la idea de que 1,5 st es un umbral para la percepción de diferencias tonales, de modo que por abajo de $1 \mathrm{st}$ es poco probable percibir una diferencia y por encima de 2 es muy probable; cf. los comentarios y referencias de Martínez Celdrán y Fernández Planas (2003). Murrieta (en preparación) está llevando a cabo una serie de experimentos perceptuales con datos y hablantes de español mexicano a partir de diferencias semitonales, y los resultados preliminares apuntan en la misma dirección. Para una discusión más general acerca de las escalas de frecuencia, véase Hermes y van Gestel (1991).

${ }^{7}$ Además del rango, puede emplearse la sincronización del pico tonal, duración de dominios como sílabas o pies, o el esfuerzo articulatorio en las vocales (Bauman et al., 2007). «Thus, the Effort Code can be realised by speakers to express contrastive focus through pitch range and duration, but they can also resort to either peak height or peak delay as an alternative to pitch range» (Vanrell et al., 2013:196).
} 
cambio, se activan de manera significativa en un amplio número de casos y ocupan un lugar más elevado en la jerarquía de restricciones, en la medida en que afectan de manera más estable a la diferenciación de uno y otro tipo de enunciados. El código de tamaño también está detrás de la altura tonal y tipo de tono de juntura final, representable por la aceptación de ALIGN (L/l, der) por los enunciados representativos y de ALIGN (H/l, der) por los directivos. Dado que las peticiones de mayor tamaño son preferentemente acogidas por las realizaciones directivas, puede caber una restricción general sobre el tamaño (SIZE) o sobre la mayor altura general del registro (REGISTER SIZE).

Por su parte, INTENSITY $/ \omega_{\mathrm{PNS}}$ e INTENSITY $/ \omega_{\mathrm{N}}$ son manifestaciones interesantes del código de producción, en la medida en que se asocian a diferentes fases secuenciales: los enunciados representativos tienden a concentrar más energía en el material prenuclear; más claramente todavía, los enunciados directivos introducen más energía en el material tonemático. Una de cada cinco veces los pares directivos tienen inicios absolutos significativamente mayores que sus contrapartes representativas. Si el correlato es alinear un tono de juntura inicial, puede asignarse una restricción de buena formación, HAVE BOUNDARY $\left({ }_{1} T\right)$, que pide la presencia de un tono de juntura inicial, de tal modo que solo lo cumplirían los directivos con diferencias significativas. Otra forma es por medio de restricciones de alineamiento: ALIGN (L/l, izq) sería respetada por los enunciados representativos, y ALIGN (H/l, izq) por algunos de los directivos, pues a fin de cuentas es una restricción que ocupa un lugar modesto. Cualquier solución formal sería manifestación del código de esfuerzo, pues los comienzos altos llaman la atención sobre el carácter peculiar del enunciado, lo que ocurre solo en algunos de los directivos.

Otras restricciones en juego son también de buena formación: NO RISE $/ \sigma_{\mathrm{PN} 1}$ es infringida siempre por ambos tipos de enunciados, por lo que su alcance es muy general pero no tiene valor diferenciador. Por su parte, NO RISE $/ \sigma_{N}$ es infringida en bastantes casos por los enunciados directivos y NO FALL/ $\sigma_{\mathrm{N}}$ por los representativos. También HAVE NUC(son) es una restricción de buena formación, en la medida en que se espera que los núcleos silábicos sean sonoros y no sordos ( $c f$. Ladefoged y Maddieson, 1996); esta restricción es infringida por los enunciados aseverativos del español mexicano central, pero respetada por los directivos, pues la inmensa mayoría de casos de ensordecimiento se concentra en los primeros ${ }^{8}$.

8 Por último, los enunciados representativos tienen siempre un L en el acento nuclear, frecuentemente $\mathrm{L}^{*}$ y ocasionalmente un $\mathrm{L}$ alineado en $\mathrm{L}+\mathrm{H}^{*}$; esta segunda solución aparece muchas veces con los enunciados directivos. Una forma preliminar de describir el hecho es por medio de dos restricciones, una de asociación, $\mathrm{L}^{*} \rightarrow \mathrm{TBU}\left(\sigma_{\mathrm{N}}{ }^{*}\right)$, respetada por los enunciados representativos e infringida por los directivos, y otra de alineamiento, $\operatorname{ALIGN}\left(\mathrm{L} / \sigma_{\mathrm{N}}, \mathrm{izq}\right)$, respetada por ambos tipos de enunciados, pero en especial por los directivos. 


\begin{tabular}{|c|c|c|}
\hline Pista fonética & Generalización sobre los resultados & Posible restricción \\
\hline $\begin{array}{l}\text { Comparación de } \\
\text { los promedios } \\
\text { de la pendiente } \\
a \text { en el modelo } \\
\text { lineal }\end{array}$ & $\begin{array}{l}\text { La diferencia entre Rep y Dir es mayor en el caso de la } \\
\text { frecuencia ( } 9,6 \text { puntos de diferencia, }-6,5 \text { en Rep, es } \\
\text { decir, notoriamente descendente, y } 3,1 \text { en Dir, } \\
\text { medianamente ascendente) que en el de la intensidad } \\
\text { (-1,4 para Rep y }-0,5 \text { para Dir) y la duración ( } 0,2 \text { para } \\
\text { Rep y } 1,1 \text { para Dir) }\end{array}$ & \multirow{4}{*}{$\begin{array}{l}\text { DOWNSTEP (Rep) } \\
\text { *DOWNSTEP (Dir) } \\
\text { (Código de producción) }\end{array}$} \\
\hline $\begin{array}{l}\text { Coeficiente } b \text { en } \\
\text { la ecuación } \\
\text { lineal }\end{array}$ & $\begin{array}{l}\text { En el 83,3\% de las comparaciones, b es mayor en los } \\
\text { enunciados representativos (Rep }>\text { Dir) }\end{array}$ & \\
\hline $\begin{array}{l}\text { Grado de ajuste } \\
\text { en el modelo } \\
\text { lineal }\end{array}$ & $\begin{array}{l}\text { El promedio siempre es mayor para los enunciados } \\
\text { Rep que para los Dir }(0,468 \text { vs. } 0,169 \text { en cuanto a la } \\
\text { frecuencia; } 0,722 \text { vs. } 0,424 \text { para la intensidad; } \\
0,095 \text { vs. } 0,050 \text { respecto a la duración) }\end{array}$ & \\
\hline $\begin{array}{l}\text { Mejoras en los } \\
\text { ajustes al pasar } \\
\text { del modelo } \\
\text { lineal al cúbico }\end{array}$ & $\begin{array}{l}\text { La mejora siempre es menor con los enunciados Rep } \\
\text { que con los enunciados Dir }(0,354 \text { vs. } 0,598 \text { en el caso } \\
\text { de la frecuencia; } 0,149 \text { vs. } 0,221 \text { para la intensidad; } \\
0,271 \text { vs. } 0,454 \text { con la duración) }\end{array}$ & \\
\hline
\end{tabular}

Tabla 2. Diferencias en pistas fonéticas globales y posible restricción afectada

Todas las observaciones fonéticas globales puede reducirse a una única restricción global, a la que se ha llamado DOWNSTEP. Esta restricción pide desarrollar un patrón declinante a lo largo de toda la frase entonativa ( $c f$. Kügler, 2011 y ms.). Comparativamente, la restricción es respetada muchas más veces por los enunciados representativos que por los directivos, como queda atestiguado por a) la pendiente lineal promedio, descendente en los enunciados representativos y ascendente en los directivos; b) el hecho de que la $b$ de la ecuación lineal sea normalmente más elevada en los enunciados representativos, pues dado que $b$ es el punto de corte en el eje $y$, la solución representativa corta más arriba debido precisamente a la mayor pendiente de la línea; c) los enunciados representativos satisfacen mejor las ecuaciones lineales en lo que toca al $\left.\mathrm{F}_{0} ; d\right)$ por lo mismo la mejora es menor para los aseverativos en los modelos cúbicos, pues quedaban mejor satisfechos que los directivos en el modelo lineal.

Una cuestión adicional es en qué medida DOWNSTEP permite interpretar correctamente los patrones de intensidad y de duración, y las relaciones entre los tres parámetros. Respecto a la pendiente $a$, la intensidad desciende más en los enunciados aseverativos que en los directivos (como es esperable), y la duración asciende un poco más en los directivos (lo que también es esperable): ambos hechos fonéticos son compatibles con un mayor cumplimiento de DOWNSTEP por parte de los enunciados aseverativos. Lo mismo puede decirse del hecho del mejor ajuste lineal de intensidad y duración con los enunciados representativos; desde luego, el ajuste es mucho mayor con la intensidad, que en sí misma queda bastante bien expuesta con las ecuaciones lineales, a diferencia de la frecuencia y la duración'. En suma, el sustento fonético para

${ }^{9}$ Este mismo hecho explica por qué el grado de mejora de la intensidad en el modelo cúbico frente al lineal sea siempre menor que el de la frecuencia o la duración para cualquier tipo de enunciado, debido a la mayor bondad de su modelo lineal; en cualquier caso, tanto intensidad como duración experimentan mayores mejoras en el modelo cúbico de los enunciados directivos, con toda probabilidad porque los modelos lineales respectivos son mejores en los enunciados aseverativos. 
DOWNSTEP se reafirma al considerar también duración e intensidad, en añadidura a la frecuencia ${ }^{10}$. La tabla 3 resume lo expuesto hasta el momento.

\begin{tabular}{|c|c|c|c|c|c|c|c|c|c|c|c|c|c|c|c|}
\hline & 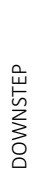 & 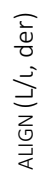 & 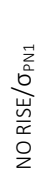 & 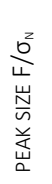 & 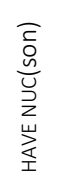 & 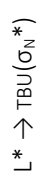 & 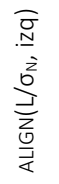 & $\begin{array}{l}\frac{a^{2}}{2} \\
\frac{1}{x} \\
\frac{1}{2}\end{array}$ & 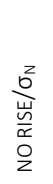 & 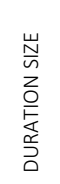 & 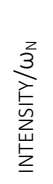 & 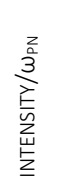 & 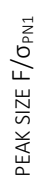 & 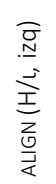 & 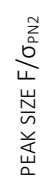 \\
\hline Rep & $\bar{~} \bar{J}$ & $\bar{l}$ & * & * & * & $\bar{l}$ & F* & * & $\bar{~} \bar{J}$ & * & * & 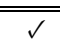 & * & * & "* \\
\hline Dir & $*$ & $*$ & $*$ & $\checkmark$ & $\checkmark$ & * & $\checkmark$ & $\checkmark$ & $*$ & $\checkmark$ & $\checkmark$ & * & $\checkmark$ & $\checkmark$ & $\checkmark$ \\
\hline
\end{tabular}

Tabla 3. Descripción fonético-fonológica de las diferencias entre dos tipos de enunciados (mayor importancia a la izquierda)

Las restricciones de la tabla 3 tienen un fuerte contenido fonético. Los enunciados aseverativos y sus pares directivos interrogativos se presentan como dos variantes (o candidatos) posibles, que tienden a estar asociados a una serie de variables independientes (las restricciones), algunas de las cuales se comportan de manera cuasicategórica y otras en forma claramente estocástica. Más allá de las posibles limitaciones, es claro su carácter regulativo, pues describen la relación del proceso dinámico con las categorías vinculadas y emplean poco contexto para hacerlo. Las tablas referidos a las propiedades locales y globales muestran, ante todo, diferencias no categóricas. Se trata de tendencias no aleatorias, sino ordenadas y representables desde modelos como la optimidad desde el punto de vista fonológico o la estadística multivariable, desde el punto de vista cuantitativo. Ambos aspectos son doblemente importantes, pues la aparición de ciertos patrones está además condicionada por la variación estilística y social, además de por ciertos parámetros lingüísticos.

\section{RASGOS EN ENUNCIADOS EXPRESIVOS: UN PROBLEMA CONSTITUTIVO}

La expresividad puede detectarse por la presencia de ciertas pistas fónicas, como el registro general más elevado (Gussenhoven, 2004), la mayor altura inicial, la mayor expansión de las prominencias, un mayor nivel de intensidad y la modificación de la duración normal de las sílabas. Existe gran cantidad de actos diferentes que pueden funcionar como subtipos expresivos (Martín Butragueño, 2014d). Una de las principales conclusiones de un trabajo exploratorio con diez informantes (2014b) es que el subtipo expresivo está ligado al menos a tres parámetros pragmáticos, que tienden a correlacionar con tres parámetros prosódicos, considerando que el pico inicial es casi siempre ascendente y que no distingue unos subtipos de otros: a) la valencia positiva o negativa del enunciado se manifiesta especialmente en el pico prenuclear, siendo los acentos descendentes los más característicamente negativos; b) el énfasis de la expresión (o magnitud de la fuerza ilocutiva) se muestra particularmente en el acento

${ }^{10}$ No se considera aquí cómo representar las diferencias de comportamiento entre los tres parámetros, como la mayor linealidad de la intensidad o los picos diferidos en la frecuencia prenuclear. 
nuclear, siendo los más fuertes los que se realizan como un acento ascendente; c) el carácter más o menos asertivo (o contundente) del enunciado se expresa ante todo a través del tono de juntura, de modo que las junturas bajas son más cerradas y las sostenidas lo son menos. Ahora bien, la combinación de estos aspectos permite disponer de una taxonomía de combinaciones. Dada la gran diversidad de planteamientos pragmáticos posibles, y ante la dificultad de sistematizar las realizaciones prosódicas obtenidas en relación a los subtipos expresivos explorados, se propuso la hipótesis de que los prototipos del desánimo y el ánimo (para la base psicológica véase Caballero, 2011), mencionados en adelante como $\alpha$ y $\beta$, se encontrarían en los extremos de la taxonomía, con muchos tipos intermedios entre ambos. Tales prototipos pueden describirse prosódicamente a través de una serie de restricciones; el cumplimiento parcial de éstas da lugar a números subtipos que combinan diferentes agregados prosódicos. El efecto de las principales restricciones puestas en juego se muestra en la tabla 4.

\begin{tabular}{|c||c:c:c|c:c|}
\hline & NO RISE/ $\omega_{\mathrm{PNI}}$ & NO RISE/ $\sigma_{\mathrm{N}}$ & ALIGN(M/l, der) & NO FALL $/ \omega_{\mathrm{PNF}}$ & ALIGN(L/1, der) \\
\hline \hline$\alpha$ 'desánimo' & & & & $*$ & $*$ \\
\hline$\beta$ 'ánimo' & $*$ & $*$ & $*$ & & \\
\hline
\end{tabular}

Tabla 4. Jerarquía de restricciones para $\alpha$ 'desánimo' y $\beta$ 'ánimo’

Las tres restricciones que mencionan el movimiento tonal de ascenso y descenso son de buena formación, mientras que las otras dos son de alineamiento. Así, NO FALL $/ \omega_{\mathrm{PNF}}$ prohíbe el contorno HL en el dominio $\omega_{\mathrm{PNF}}$, es decir, el contorno descendente en la última palabra prosódica adscrita al material prenuclear; esta restricción se infringe cuando se comunica desánimo. Se presentan las jerarquías pertinentes para dar como ganadores a uno u otro prototipo, pero dado el carácter de prototipos, en ningún caso se marca un efecto crucial (!) por parte de una restricción. Lo más interesante es que pueden describirse otros tipos intermedios a través del respeto o infracción a cada una de estas restricciones, de modo que se quede más cerca o más lejos de cada uno de los prototipos establecidos ${ }^{11}$.

Para poner a prueba en particular la restricción NO RISE $/ \sigma_{\mathrm{N}}$ sobre la sílaba nuclear, se trabajó (cf. 2014d para el análisis en detalle) con una muestra de 720 enunciados expresivos procedentes de entrevistas sociolingüísticas con 36 informantes, parte del Corpus sociolingüistico de la ciudad de México (Martín Butragueño y Lastra, 2011, 2012 y en preparación). La tabla 5 muestra los acentos tonales y la 6 los prototipos.

${ }^{11}$ Tal como se expone en el trabajo de (2014b), existen otras restricciones en juego, que dan constitución específica a otros aspectos pragmáticos y prosódicas; no me referiré ahora a ellas. 


\begin{tabular}{|c|c|c|}
\hline Acento & $\begin{array}{c}\text { Frecuencia } \\
\text { absoluta }\end{array}$ & Porcentaje \\
\hline $\mathrm{L}+{ }_{i} \mathrm{H}^{*}$ & 417 & $57,9 \%$ \\
\hline $\mathrm{L}+\mathrm{H}^{*}$ & 182 & $25,3 \%$ \\
\hline$! \mathrm{H}^{*}$ & 59 & $8,2 \%$ \\
\hline $\mathrm{H}^{*}$ & 40 & $5,6 \%$ \\
\hline $\mathrm{H}+\mathrm{L}^{*}$ & 10 & $1,4 \%$ \\
\hline $\mathrm{H}+\mathrm{L}^{*}$ & 7 & $1,0 \%$ \\
\hline L* & 5 & $0,7 \%$ \\
\hline Total $=$ & 720 & $100 \%$ \\
\hline
\end{tabular}

Tabla 5. Resultados por acento tonal (fonético-fonológico)

Los bitonos ascendente son los más abundantes; entre $\mathrm{L}+{ }_{i} \mathrm{H}^{*}$ y $\mathrm{L}+\mathrm{H}^{*}$ suman el $83,2 \%$ de los casos. Esto es interesante, pues en una muestra general de enunciados aseverativos (Martín Butragueño, 2011), la proporción de bitonos ascendentes era de $62,2 \%$. Es decir, la muestra de actos expresivos se encuentra 21 puntos por arriba. A efectos estadísticos, se reagrupan los acentos tonales en cuatro variantes. Las ascendentes son compatibles con el prototipo $\beta$ e infringen la restricción NO RISE $/ \sigma_{\mathrm{N}}$. Las no ascendentes entran en la esfera de $\alpha$ y respetan la restricción (tabla 6).

\begin{tabular}{|c|c|c|c|c|}
\hline NO RISE/ $\sigma_{N}$ & Prototipo & Variante & $\begin{array}{c}\text { Frecuencia } \\
\text { absoluta }\end{array}$ & Porcentaje \\
\hline \multirow[t]{2}{*}{ Se infringe: } & $\beta$ & $\mathrm{L}+{ } \mathrm{H}^{*}$ & 417 & $57,9 \%$ \\
\hline & $\beta$ & $\mathrm{L}+\mathrm{H}^{*}$ & 182 & $25,3 \%$ \\
\hline \multirow{3}{*}{ Se respeta: } & $\alpha$ & $(!) \mathrm{H}^{*}$ & 99 & $13,8 \%$ \\
\hline & $\alpha$ & $(\mathrm{H}+!) \mathrm{L}^{*}$ & 22 & $3,1 \%$ \\
\hline & & Total $=$ & 720 & $100 \%$ \\
\hline
\end{tabular}

Tabla 6. Resultados por variante

La figura 1 muestran un ejemplo de $\mathrm{L}+{ }_{i} \mathrm{H}^{*}$ en la sílaba nuclear ${ }^{12}$.

${ }^{12} \mathrm{La}$ línea continua es el $\mathrm{F}_{0}$ y la discontinua la intensidad. En los ejemplos, el primer dígito es «1» para estudios bajos (primaria o menos), «2» para estudios medios (bachillerato como máximo), «3» para estudios altos (universitarios); el segundo dígito es edad «1»(20-34), «2» (35-54) о «3» (55 en adelante); la letra es «H», hombre, o «M», mujer; se anota también el número de entrevista. 


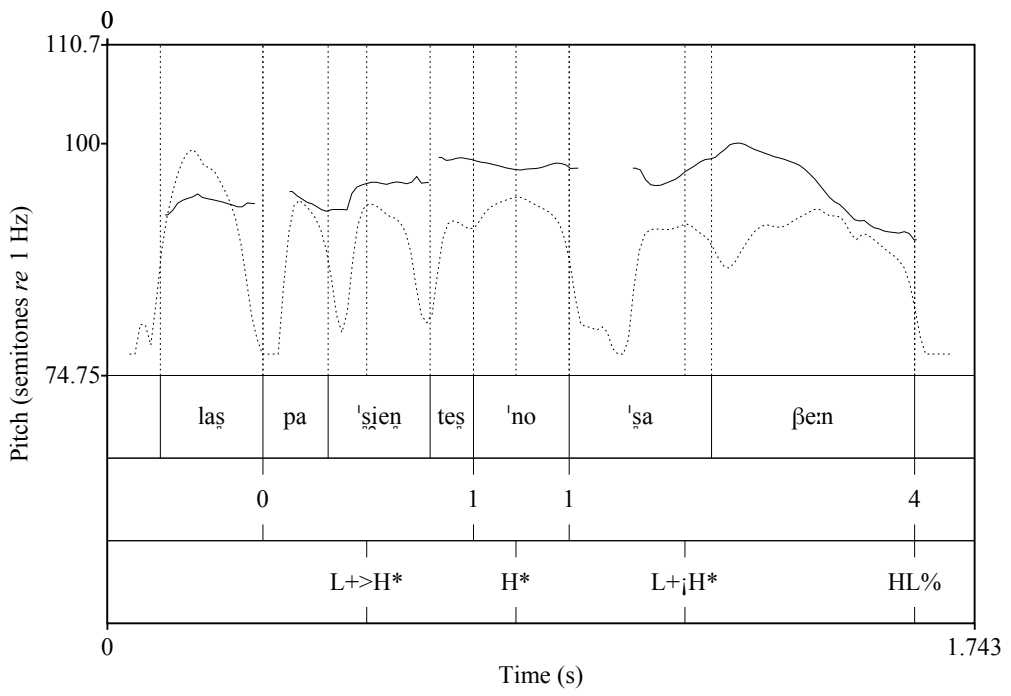

Figura 1. ¡Las pacientes no saben! (31M, ent. 252)

Las tablas 7 y 8 introducen los resultados significativos para $\mathrm{L}+{ }_{i} \mathrm{H}^{*}$. Desde el punto de vista fónico, el acento se ve favorecido por cualquier duración superior a 100 $\mathrm{ms}$, lo que es coherente con la idea de un acento tonal con una elevación prominente ${ }^{13}$.

\begin{tabular}{|c|r|r|c|r|r|r|c|}
\hline Factor & \multicolumn{1}{|c|}{$F / N$} & $\%$ & $P$ & Factor & $F / N$ & $\%$ & $P$ \\
\hline \multicolumn{6}{|c|}{ a) Tiempo previo al pico } & \multicolumn{2}{|c|}{ b) Ámbito del énfasis expresivo } \\
\hline $300-686 \mathrm{~ms}$ & $44 / 62$ & 71.0 & 0.664 & Local & $202 / 275$ & 73.5 & 0,663 \\
$100-199 \mathrm{~ms}$ & $239 / 374$ & 63.9 & 0.561 & Global & $215 / 445$ & 48.3 & 0,397 \\
$200-299 \mathrm{~ms}$ & $97 / 153$ & 63.4 & 0.547 & Rango $=26$ & & & \\
$0-99 \mathrm{~ms}$ & $37 / 131$ & 28.2 & 0.223 & & & & \\
Rango $=41$ & & & \multicolumn{6}{|c|}{} & & & \\
\hline
\end{tabular}

Tabla 7. Grupos de factores lingüísticos significativos para $\mathrm{L}+{ }_{i} \mathrm{H}^{*}$

El pico promedio se presenta cuando ha transcurrido aproximadamente el $80 \%$ de la sílaba (el 78,7\%, exactamente), lo que sugiere una realización adelantada del pico, representable como $\mathrm{L}+<_{i} \mathrm{H}^{*}$. La idea de un pico temprano como realización usual del pico expresivo supondría que no solo la altura, sino también el alineamiento, resultaría diferente de los picos nucleares de enunciados no expresivos. Tratándose de una diferencia pragmática, su presencia parcialmente convencional desempeñaría un papel intermedio entre el detalle fonético y la categoricidad fonológica. El segundo grupo de factores significativo, el carácter global o local del énfasis expresivo, es pragmático. Es el doble de probable que $\mathrm{L}+{ }_{i} \mathrm{H}^{*}$ esté asociado a un énfasis local $(\mathrm{p}=0,663 \text { vs. } 0,397)^{14}$.

13 No siempre las cosas así, pues puede haber ascensos prominentes con duraciones no proporcionales, lo que da lugar a elevadas velocidades de ascenso, como ocurre con algunas personas en el centro de México (Martín Butragueño, 2014c).

14 Aunque esta asociación entre énfasis estrecho y acentos tonales elevados es esperable, el promedio de altura de $\mathrm{L}+{ }_{i} \mathrm{H}^{*}$ es virtualmente idéntico cuando la expresividad es global $(6,0$ st $)$ que 
No sorprende demasiado la vinculación del acento $\mathrm{L}+{ }_{i} \mathrm{H}^{*}$ con el género (para las mujeres, $\mathrm{p}=0,541$, por 0,459 de los hombres), pues diferentes trabajos relacionados con la prominencia nuclear (por ejemplo Martín Butragueño, 2014c) muestran reiteradamente el mismo resultado: los hombres tienden a mostrar movimientos tonales nucleares más pronunciados.

La variante $\mathrm{L}+\mathrm{H}^{*}$ también infringe NO RISE $/ \sigma_{\mathrm{N}}$. La tabla 8 muestra los hechos relevantes desde un punto de vista estadístico, que fueron solo lingüísticos.

\begin{tabular}{|c|c|c|c|c|c|c|c|}
\hline Factor & $F / N$ & $\%$ & $P$ & Factor & $F / N$ & $\%$ & $P$ \\
\hline \multicolumn{4}{|c|}{ a) Velocidad de ascenso } & \multicolumn{4}{|c|}{ c) Cortesia } \\
\hline$<0.05 \mathrm{st} / \mathrm{ms}$ & $178 / 630$ & 28.3 & 0.569 & Salvaguarda positiva & $8 / 18$ & 44,4 & 0,723 \\
\hline$>0.05 \mathrm{st} / \mathrm{ms}$ & $4 / 90$ & 4.4 & 0.125 & Salvaguarda negativa & $7 / 30$ & 23,3 & 0,473 \\
\hline \multirow[t]{2}{*}{ Rango $=44$} & & & & Amenaza & $7 / 53$ & 13,2 & 0,278 \\
\hline & & & & $\begin{array}{r}\text { Casos neutros } \\
\text { Rango }=44\end{array}$ & $160 / 619$ & 25,8 & 0,515 \\
\hline \multicolumn{4}{|c|}{ b) Localidad } & \multicolumn{4}{|c|}{ d) Porcentaje de la sílaba en el pico } \\
\hline Global & $129 / 445$ & 29.0 & 0.559 & $30,0-59,9 \%$ & $46 / 137$ & 33,6 & 0,617 \\
\hline \multirow{4}{*}{$\begin{array}{r}\text { Local } \\
\text { Rango }=15\end{array}$} & $53 / 27$ & 19.3 & 0.405 & $60,0-89,9 \%$ & $72 / 293$ & 24,6 & 0,498 \\
\hline & & & & $90,0-100 \%$ & $56 / 243$ & 23,0 & 0,465 \\
\hline & & & & $0,0 \%-29,9 \%$ & $8 / 47$ & 17,0 & 0,352 \\
\hline & & & & Rango & & & \\
\hline
\end{tabular}

Razón de verosimilitud $=-377,396$, sign. $=0,024$, input $=0,222$

Tabla 8. Grupos de factores lingüísticos significativos para $\mathrm{L}+\mathrm{H}^{*}$

La velocidad de ascenso es notable en el modelo $($ rango $=44)$, pues si la melodía transcurre por abajo de $0,05 \mathrm{st} / \mathrm{ms}$, la probabilidad alcanza 0.569 , lo que cuadra bien con la idea de expresividad moderada. Tampoco en el caso de $\mathrm{L}+\mathrm{H}^{*}$ el pico tonal se alcanza necesariamente al final de la sílaba nuclear. La mayor probabilidad $(0,617)$ es para el segmento de 30,0 a 59,9\%, en la parte media de la sílaba nuclear. Si se suman los picos que surgen antes del $90 \%$ del transcurso de la sílaba (126 casos), resulta que 69,2\% de $\mathrm{L}+\mathrm{H}^{*}$ culminan antes del final de la sílaba, lo que confirma que el pico temprano está vinculado a la expresividad. Con $\mathrm{L}+\mathrm{H}^{*}$, el tiempo promedio previo al pico es $167,7 \mathrm{~ms}$, y la media posterior es $64.2 \mathrm{~ms}$. Quizá pueda hablarse de un acento expresivo con un $\mathrm{L}+<\mathrm{H}^{*}$ temprano de la misma forma que con el provisional $\mathrm{L}+<_{j} \mathrm{H}^{*}$. El ámbito del énfasis expresivo y la cortesía dan contenido pragmático a $\mathrm{L}+\mathrm{H}^{*}$. La expresividad global $(\mathrm{p}=0,559)$ favorece la presencia de la variante, estableciéndose así un patrón complementario al de $\mathrm{L}+{ }_{i} \mathrm{H}^{*}$. La presencia de la prominencia moderada en sílaba nuclear está asociada a la salvaguarda positiva, no a la negativa ni a la amenaza: $\mathrm{L}+\mathrm{H}^{*}$ es favorecido por la cortesía que subraya la pertenencia al grupo.

Las dos variantes restantes, (!) $\mathrm{H}^{*}$ y $(\mathrm{H}+!) \mathrm{L}^{*}$, respetan NO RISE $/ \sigma_{\mathrm{N}}$, al no presentar ascenso en la sílaba nuclear. La figura 2 presenta un ejemplo de $\mathrm{H}^{*}$.

cuando es local sobre el acento (6,1 st). De los 202 casos de prominencia local, 124 (el 61,3\%) corresponden claramente a focos estrechos. Los restantes requieren un análisis pragmático más detallado, pero sugieren un fuerte grado de involucramiento con el enunciado. 


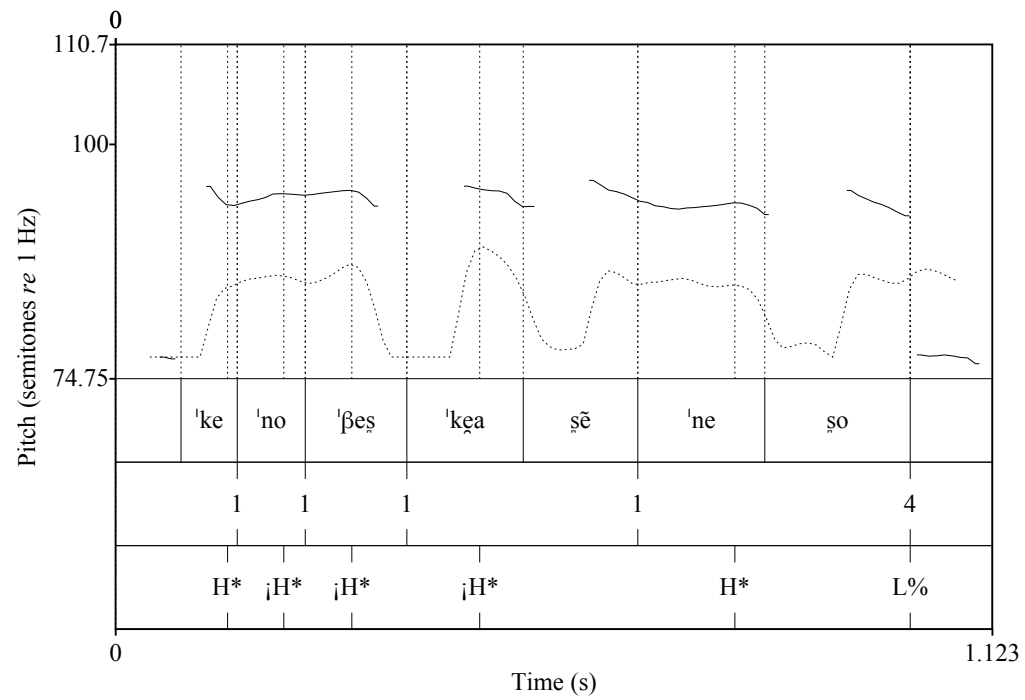

Figura 2. ¡¿Qué no ves que hacen eso?! (22M, ent. 274)

Los 99 ejemplos de (!)H* muestran solo dos grupos de factores lingüísticos significativos: el tiempo transcurrido antes de la presencia del pico (si es menor a $100 \mathrm{~ms}, \mathrm{p}=0.759$ ) y el tipo de ámbito de la prominencia expresiva (cuando es global, $\mathrm{p}=0.611)$. No hubo variables sociolingüísticas de peso.

Es interesante comparar el comportamiento de las tres variantes tonales no descendentes, que resultan estar muy ordenadas:

$$
\begin{aligned}
& \text { a. } \mathrm{L}+{ }_{i} \mathrm{H}^{*}(242.4)>\mathrm{L}+\mathrm{H}^{*}(231.8)>(!) \mathrm{H}^{*}(211.7) \text { [Duración de la sílaba en ms]. } \\
& \text { b. } \mathrm{L}+{ }_{i} \mathrm{H}^{*}(190.8)>\mathrm{L}+\mathrm{H}^{*}(167.7)>(!) \mathrm{H}^{*}(128.3) \text { [Material previo al pico, en ms]. } \\
& \text { c. } \mathrm{L}+\mathrm{H}^{*}(80.4)>\mathrm{L}+\mathrm{H}^{*}(73.4)>(!) \mathrm{H}^{*}(59.9) \text { [Porcentaje transcurrido antes del pico, en \%]. }
\end{aligned}
$$

No hubo factores lingüísticos significativos para $(\mathrm{H}+i) \mathrm{L}^{*}$, aunque ciertamente se trata de muy pocos casos (solo 22). El género es de nuevo significativo, pero son las mujeres quienes favorecen la variante $(\mathrm{p}=0,623$, vs. 0,377 en hombres). El patrón es complementario al obtenido con $\mathrm{L}+{ }_{i} \mathrm{H}^{*}$.

\section{DISCUSIÓN Y CONCLUSIONES}

Queda mucho por hacer sobre los actos de habla dentro de una PBU. Una estrategia es partir de datos más controlados para después abrir el análisis a los datos más naturales y realistas posibles. La exposición ha contrastado pares mínimos leídos para las diferencias entre enunciados aseverativos y directivos interrogativos absolutos, y entrevistas sociolingüísticas para caracterizar algunos tipos de enunciados expresivos.

Los fenómenos prosódicos pueden considerarse regulaciones, constituciones o instrucciones según el tipo de alternancia que presenten las variantes en juego y según la cantidad de contexto y la generalidad o circunstancialidad con que puedan describirse. El tipo de descripción es consecuencia del método empleado y de la naturaleza misma 
del fenómeno. El par representación-pregunta se trata como una regulación porque son pares mínimos aislados de todo contexto y permiten una formulación donde cada uno de los miembros del par se presenta como una variante de una variable dependiente, el tipo de enunciado. Y cada restricción es un grupo de dos factores (respeto o infracción). Así, los datos admiten un tratamiento multivariable, incluso probabilístico, sea que se vea desde un punto de vista variacionista o desde una perspectiva optimal estocástica, como muchas otras variables fónicas segmentales y numerosas variables sintácticas.

Los enunciados expresivos han recibido un tratamiento constitutivo, cercano al de muchos procesos léxico-semánticos. Las diferentes soluciones (del todo o de las partes) se describen a partir de una serie de grupos de factores (las mismas restricciones), pero no se pretende que los enunciados sean pares mínimos, por lo que la relación entre ellos y sus descriptores debe incorporar mayor cantidad de contexto, para que se pueda saber qué valor adquieren según qué circunstancia general.

Las restricciones optimales empleadas dan forma a regulaciones y constituciones, en la medida en que permiten comparar unos actos de habla con otros, y observar el diferente efecto de cada restricción según el objetivo pragmático perseguido. El enfoque es flexible para adaptarse a datos que no se comportan de forma categórica ${ }^{15}$. Con un núcleo pequeño de recursos y posibilidades es posible, al explotarlos y combinarlos de múltiples maneras, comunicar sentidos y realizar acciones lingüísticas virtualmente innumerables. Es necesario trabajar con versiones estocásticas de la teoría de la optimidad, perspectiva para la que existe una gran variedad de propuestas. La versión seguida para las expresiones trabaja por lo general con el mismo algoritmo de regresión logística que es común en numerosos trabajos de variación lingüística ${ }^{16}$. En cuanto a las instrucciones, no es que un modelo de jerarquía de restricciones no sea aplicable; seguramente lo es, pero es verosímil que muchos problemas interesantes queden fuera de su alcance.

Una PBU tiene que conjugar todos estos elementos, presentes de diferentes formas en regulaciones, constituciones e instrucciones, por lo que suponen en términos metodológicos y, sobre todo, por lo que implican en cuanto a la naturaleza de los datos y a la manera en que desafían al desarrollo actual de la prosodia. Se ha insistido en la necesidad de que una PBU estudie materiales realistas desde un punto de vista empírico. La fundamentación del uso no implica solo el análisis de una cantidad significativa de materiales en fragmentos de habla espontánea. Implica también el cuestionamiento de las relaciones fonético-fonológicas con diferentes tipos de enunciados y el trabajo con situaciones testigo que permitan entender cómo los hablantes construyen su prosodia a partir de diferentes procesos. Varios ejemplos específicos muy distintos pueden ser $a$ ) los enfoques interaccionistas de adquisición y desarrollo lingüístico; b) las ventajas buscadas y obtenidas por los hablantes en conversaciones cotidianas; c) las situaciones

\footnotetext{
${ }^{15}$ Otra cuestión es que se haya presentado una formulación muy preliminar de las restricciones necesarias para describir adecuadamente la prosodia de los actos de habla del español mexicano.

${ }^{16}$ Cuando menos para abordar los aspectos relevantes en los datos base, sin que haya parecido necesario transferir ahora el algoritmo en todo su detalle a cada restricción. Si un acento nuclear ascendente $\mathrm{T}^{*}$ aparece con una probabilidad $p$ en ciertos enunciados, esa misma $p$ es la de infringir NO RISE.
} 
de contacto dialectal en situaciones migratorias o fronterizas y el contacto lingüístico en general; $d$ ) la construcción de identidades por parte de grupos de diferentes tamaños; $e$ ) los fenómenos de prosodificación, en términos de los pasos que sigue y de los materiales que aprovecha.

\section{REFERENCIAS BIBLIOGRÁFICAS}

Aguilar Ruiz, M. C. (2012): La entonación del habla infantil de la ciudad de Puebla, tesis de licenciatura, Puebla, Benemérita Universidad Autónoma de Puebla.

AMPER. Atlas Multimédia Prosodique de l'Espace Roman [en línea]: $<$ http://stel.ub.edu/labfon/amper/cast/index.html $>$.

Baumann, S., J. Johannes Becker, M. Grice y D. Mücke (2007): «Tonal and articulatory marking of focus in German», en Trouvain, J. y W. J. Barry, eds., 16th International Congress of Phonetic Sciences (ICPhS16), Saarbrücken, Dudweiler, Pirrot GmbH, 1029-1032.

Boersma, P. y B. HAYes (2001): «Empirical tests of the Gradual Learning Algorithm», Linguistic Inquiry, vol. 32, 45-86, <http://dx.doi.org/10.1162/002438901554586>.

Borràs-COMES, J., M. VANRELl y P. PRIETO (2014): «The role of pitch range in establishing intonational contrasts», Journal of the International Phonetic Association, vol. 44, 1, 1-20, <http://dx.doi.org/10.1017/s0025100313000303>.

ByBee, J. L. (2001): Phonology and Language Use, Cambridge, CUP, $<$ http://dx.doi.org/10.1017/cbo9780511612886>.

ByBEe, J. L. (2008): «Formal universals as emergent phenomena: the origins of structure preservation», en Good, J., ed., Linguistic Universals and Language Change, Oxford, OUP, 108-121, $<$ http://dx.doi.org/10.1093/acprof:oso/9780199298495.003.0005>.

Bybee, J. L. y P. Hopper, eds., (2001): Frequency and the Emergence of Linguistic Structure, Ámsterdam-Filadelfia, John Benjamins.

Caballero Meneses, J. A. (2011): Contribuciones biológicas, psicofisiológicas y culturales a la prosodia del lenguaje, tesis doctoral, México, UNAM.

Coetzee, A. W. (2009): "An integrated grammatical/non-grammatical model of phonological variation», en Kang, Y.-S. et al., eds., Current Issues in Linguistic Interfaces, Seúl, Hankookmunhwasa, vol. 2, 267-294.

Coetzee, A. W. y S. KAWAHARA (2013): «Frequency biases in phonological variation», Natural Language and Linguistic Theory, vol. 31, 1, 47-89,

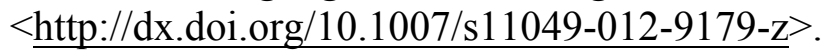

DE JONG, K. (1995): «The supraglottal articulation of prominence in English: Linguistic stress as localized hyperarticulation», Journal of the Acoustical Society of America, vol. 97, 491-504, <http://dx.doi.org/10.1121/1.412275>.

DíAz-CAMPOS, M. y S. COLINA (2006): "The interaction between faithfulness constraints and sociolinguistic variation: the acquisition of phonological variation in first language speakers», en Martínez-Gil, F. y S. Colina, eds., 
Optimality-Theoretic Studies in Spanish Phonology, Ámsterdam-Filadelfia, John Benjamins, 424-446, <http://dx.doi.org/10.1075/la.99.17dia $>$.

DITTMAR, N. (1996): «Descriptive and explanatory power of rules in sociolinguistics», en Singh, R., ed., Towards a Critical Sociolinguistics, Ámsterdam-Filadelfia, John Benjamins, 115-149.

ECKerT, P. (2000): Linguistic Variation as Social Practice, Oxford, Blackwell.

ECKERT, P. (2008): «Variation and the indexical field», Journal of Sociolinguistics, vol. 12, 453-476, <http://dx.doi.org/10.1111/j.1467-9841.2008.00374.x >.

Feldhausen, I. y M. VANRELl (2014): «Prosody, focus and word order in Catalan and Spanish. An Optimality Theoretic approach», en Proceedings of the 10th International Seminar on Speech Production (ISSP), 5-8 de mayo 2014, Colonia.

Gussenhoven, C. (2002): «Intonation and interpretation: Phonetics and phonology», en Bel, B. e I. Marlien, Speech Prosody 2002, Aix-en-Provence, Laboratoire Parole et Langage, 47-57.

Gussenhoven, C. (2004): The Phonology of Tone and Intonation, Cambridge, CUP, $<$ http://dx.doi.org/10.1017/cbo9780511616983 $>$.

GuY, G. (1994): «The phonology of variation», en Beals, K. et al., eds., Papers from the 30th Regional Meeting of the Chicago Linguistic Society, 2: The Parasession on Variation in Linguistic Theory, Chicago, CLS, 133-149.

GuY, G. (1997): «Violable is variable: Optimality Theory and linguistic variation», Language Variation and Change, vol. 9, 333-347, $<\underline{\mathrm{http}}$ //dx.doi.org/10.1017/s0954394500001952>.

GuY, G. (2007): «Variation and phonological theory», en Bayley, R. y C. Lucas, eds., Sociolinguistic Variation. Theories, Methods and Applications, Cambridge, CUP, 5-23, <http://dx.doi.org/10.1017/cbo9780511619496.002>.

Hermes, D. J. y J. C. VAN GeSTEL (1991): «The frequency scale of speech intonation», Journal of the Acoustical Society of America, vol. 90, 1, 97-102, $<$ http://dx.doi.org/10.1121/1.402397>.

Hidalgo, A. (1997): Entonación coloquial. Función demarcativa y unidades de habla, Valencia, Universitat de València.

Hidalgo, A. (2009): «Modalización (des)cortés y prosodia: estado de la cuestión en el ámbito hispánico», Boletín de Filología de la Universidad de Chile, vol. 44, 1, 161-195.

HidAlgO, A. (2011): «La investigación de la entonación coloquial: hacia un estado de la cuestión en el ámbito hispánico», Oralia, vol. 14, 15-45.

Hualde, J. I. y P. PRIETO (en preparación): Towards an International Prosodic Alphabet.

Kostakis, A. (2010): "Vestige theory: sociolinguistic evidence for output-output constraints», Lingua, vol. 120, 2476-2496, $<$ http://dx.doi.org/10.1016/j.lingua.2010.04.010 $>$.

KÜGLER, F. (2011): The prosodic expression of focus in typologically unrelated languages. Kumulative Habilitationsschrift, Potsdam, Universität. [en línea]: $<$ http://www.ling.uni- 
potsdam.de/ kuegler/docs/Kuegler.Habilitation.06.2011.pdf $>$

[Consulta: 31/01/2014].

KÜGLER, F. (ms.): Modeling cross-linguistic pitch-range effects for tonal implementation. [en línea]:

$<$ http://www.prosodylab.org/ chael/www/etap/abstracts/posters/kuegler.pdf $>$ [Consulta: 31/01/2014].

Labov, W. (1994): Principles of Linguistic Change, vol. 1: Internal Factors, Oxford, Blackwell.

Labov, W. (2001): Principles of Linguistic Change, vol. 2: Social Factors, Oxford, Blackwell.

Labov, W. (2010): Principles of Linguistic Change, vol. 3: Cognitive and Cultural Factors, Oxford, Wiley-Blackwell.

LADEFoged, P. e I. Maddieson (1996): The Sounds of the World's Lanaguages, Oxford, Blackwell.

Lieberman, P. (1967): Intonation, perception and language, Cambridge, MIT Press.

Martín ButragueÑo, P. (2011): «Estratificación sociolingüística de la entonación circunfleja mexicana», en P. Martín, ed., Realismo en el análisis de corpus orales. Primer coloquio de cambio y variación lingüistica, México, El Colegio de México, 93-121.

Martín Butragueño, P. (2014a): Fonología variable del español de México, vol. 1: Procesos segmentales, México, El Colegio de México.

Martín ButragueÑo, P. (2014b): «Acercamiento a la prosodia de los actos de habla expresivos. Datos del español de México», en Hernández, E. y P. Martín, eds., Variación y diversidad lingüística: hacia una teoría convergente, México, El Colegio de México, 259-349.

Martín ButragueÑo, P. (2014c): «Historia de dos medidas: contacto entonativo en la ciudad de México», conferencia plenaria en el IV Coloquio de cambio $y$ variación lingüística: contacto de lenguas y dialectos, México, Universidad Nacional Autónoma de México, 6-8 de octubre 2014.

Martín Butragueño, P. (2014d): A veces lloro mis lágrimas. Acercamiento multivariable a la prosodia de los actos de habla expresivos en el español de México, ms.

MARTín ButRagueÑo, P. (en prensa): «Prosodia fonética de enunciados representativos e interrogativos absolutos: elementos locales y globales», Estudios de Fonética Experimental.

Martín Butragueño, P. y Y. LASTRA (coords.) 2011. Corpus sociolingüistico de la ciudad de México, I: Hablantes de instrucción superior, México, El Colegio de México.

Martín Butragueño, P. y Y. LASTRA (coords.) 2012. Corpus sociolingüístico de la ciudad de México, II: Hablantes de instrucción media, México, El Colegio de México.

Martín Butragueño, P. y Y. LASTRA (coords.) (en preparación), Corpus sociolingüistico de la ciudad de México, III: Hablantes de instrucción baja, México, El Colegio de México. 
Martín Butragueño, P. y E. P. Velásquez Upegui (2014): «Prosodia basada en el uso. Proyecto para el estudio sociolingüístico del español de España y América», en Reunión del Proyecto Preseea João Pessoa, Paraíba, Brasil, 14-19 de julio 2014 [en línea]: $<$ http://preseea.linguas.net/Metodolog\%C3\%ADa.aspx $>$.

MARTínez CELDRÁN, E. (2011): «La línea melódica de la entonación declarativa e interrogativa absoluta en el español de España», en Hidalgo, A., Y. Congosto y M. Quilis, eds., El estudio de la prosodia en España en el siglo XXI: perspectivas y ámbitos, Valencia, Universitat de València, 125-140.

Martínez Celdrán, E. y A. M. Fernández Planas (2003): «Taxonomía de las estructuras entonativas de las modalidades declarativa e interogativa del español estándar peninsular según el modelo $A M$ en habla de laboratorio», en Herrera, E. y P. Martín, eds., La tonía: dimensiones fonéticas y fonológicas, México, El Colegio de México, 267-294.

MuRRIETA, L. (en preparación): Análisis experimental del umbral de percepción entonativa en el español del centro de México, tesis doctoral, México, UNAM.

OhalA, J. (1983): «Cross language use of pitch: An ethological view», Phonetica, vol. 40, 1-1, <http://dx.doi.org/10.1159/000261678>.

OHALA, J. (1984): «An ethological perspective on common cross-language utilitzation of $\mathrm{F}_{0}$ in voice», Phonetica, vol. 41, 1-16, <http://dx.doi.org/10.1159/000261706>.

OHALA, J. (1994): «The frequency code underlines the sound symbolic use of voice of pitch», en Hinton, L., J. Nichols y J. J. Ohala, Sound symbolism, Cambridge, CUP, 325-347, <http://dx.doi.org/10.1017/cbo9780511751806.022>.

Orozco, L. (2010): Estudio sociolingüístico de la cortesía en tratamientos y peticiones. Datos de Guadalajara, tesis doctoral, México, El Colegio de México.

OrozCO, L. (2012): «Propuesta de clasificación de las situaciones del ATLES en tipos de actos de habla», Seminario de Prosodia de El Colegio de México, 12 de junio 2012.

PATER, J. (2009): «Weighted constraints in generative linguistics», Cognitive Science, vol. 33, 999-1035, <http://dx.doi.org/10.1111/j.1551-6709.2009.01047.x >.

Prince, A. y P. SMOLENSKY (2004): Optimality Theory. Constraint Interaction in Generative Grammar, Oxford, Blackwell [original de 1993], http://dx.doi.org/10.1002/9780470756171.ch1.

Serrano, J. (2014): Procesos sociolingüísticos en el español de la ciudad de México. Estudio en tiempo real, tesis doctoral, México, El Colegio de México.

Tomasello, M. (2003): Constructing a Language: A Usage-based Theory of Language Acquisition, Harvard, Harvard University Press.

Vanrell, M., A. Stella, B. Gili Fivela y P. Prieto (2013): «Prosodic manifestations of the Effort Code in Catalan, Italian and Spanish Contrastive Focus», Journal of the International Phonetic Association, vol. 43, 2, 195-220, $<$ http://dx.doi.org/10.1017/s0025100313000066>.

Yule, G. (2011): Pragmatics, Oxford, OUP. 